ANNALES

POLONICI MATHEMATICI

$94.1(2008)$

\title{
Existence and uniqueness of periodic solutions for a kind of nonlinear $n$th order differential equations with delays
}

\author{
by Weiwen Shao (Beijing), Fuxing Zhang (Shaoyang) \\ and YA Li (Changsha)
}

\begin{abstract}
By applying the continuation theorem of coincidence degree theory, we establish new results on the existence and uniqueness of $2 \pi$-periodic solutions for a class of nonlinear $n$th order differential equations with delays.
\end{abstract}

1. Introduction. In this paper, we study the existence and uniqueness of $2 \pi$-periodic solutions of the nonlinear $n$th order delay differential equation

$$
x^{(n)}+\sum_{j=1}^{n-1} a_{j} x^{(j)}+g(t, x(t-\tau(t)))=p(t),
$$

where $\tau, p: \mathbb{R} \rightarrow \mathbb{R}$ and $g: \mathbb{R} \times \mathbb{R} \rightarrow \mathbb{R}$ are continuous functions, $\tau(t)$ and $p(t)$ are $2 \pi$-periodic with respect to $t, g$ is $2 \pi$-periodic in the first variable, $n \geq 2$ is an integer, and $a_{j}(j=1, \ldots, n-1)$ are constants.

During the past thirty years, there has been a great amount of work on the existence of periodic solutions for the higher-order Duffing equation

$$
x^{(2 k)}+\sum_{j=1}^{k-1} a_{j} x^{(2 j)}+(-1)^{k+1} g(t, x)=0,
$$

or

$$
x^{(2 k+1)}+\sum_{j=1}^{k-1} a_{j} x^{(2 j+1)}+g(t, x)=0 .
$$

Many of these results can be found in $[1,5,6,12-14,16]$ and the references cited therein. However, to the best of our knowledge, there exist few results on the existence and uniqueness of $2 \pi$-periodic solutions of (1.1).

2000 Mathematics Subject Classification: 34K15, 34C25.

Key words and phrases: $n$th order differential equation, periodic solution, existence, uniqueness, coincidence degree, delay.

This work was supported by the Scientific Research Fund of Hunan Provincial Natural Science Foundation of China (07JJ46001). 
The main purpose of this paper is to establish sufficient conditions for the existence and uniqueness of $2 \pi$-periodic solutions of (1.1). Our results are new and they complement previously known results. An illustrative example is given in Section 4 .

If $n$ is even, let $n=2 k$; then equation (1.1) becomes

$$
x^{(2 k)}+\sum_{j=1}^{2 k-1} a_{j} x^{(j)}+g(t, x(t-\tau(t)))=p(t) .
$$

If $n$ is odd, let $n=2 k+1$; then (1.1) becomes

$$
x^{(2 k+1)}+\sum_{j=1}^{2 k} a_{j} x^{(j)}+g(t, x(t-\tau(t)))=p(t) .
$$

For ease of exposition, throughout this paper we will adopt the following notations:

$$
\begin{aligned}
|x|_{p}=\left(\int_{0}^{2 \pi}|x(t)|^{p} d t\right)^{1 / p}, \quad|x|_{\infty}=\max _{t \in[0,2 \pi]}|x(t)|, \quad a^{+}=\max \{0, a\} \\
\|x\|=\sum_{j=0}^{n-1}\left|x^{(j)}\right|_{\infty}, \quad x^{(0)}=x \\
A_{1}=1-a_{2(k-1)}^{+}-\left|a_{2(k-2)}\right|-\cdots-\left|a_{4}\right|-a_{2}^{+}, \\
A_{2}=a_{2 k-1}-a_{2 k-3}^{+}-\left|a_{2 k-5}\right|-\cdots-\left|a_{3}\right|-a_{1}^{+}, \\
\bar{A}_{1}=1-a_{2(k-1)}^{+}-\left|a_{2(k-2)}\right|-\cdots-a_{4}^{+}-\left|a_{2}\right| \\
\bar{A}_{2}=a_{2 k-1}-a_{2 k-3}^{+}-\left|a_{2 k-5}\right|-\cdots-a_{3}^{+}-\left|a_{1}\right| \\
A_{3}=1-a_{2 k-1}^{+}-\left|a_{2 k-3}\right|-\cdots-a_{3}^{+}-\left|a_{1}\right| \\
A_{4}=a_{2 k}-a_{2 k-2}^{+}-\left|a_{2 k-4}\right|-\cdots-\left|a_{4}\right|-a_{2}^{+} \\
\bar{A}_{3}=1-a_{2 k-1}^{+}-\left|a_{2 k-3}\right|-\cdots-\left|a_{3}\right|-a_{1}^{+}, \\
\bar{A}_{4}=a_{2 k}-a_{2 k-2}^{+}-\left|a_{2 k-4}\right|-\cdots-\cdots-a_{4}^{+}-\left|a_{2}\right| .
\end{aligned}
$$

It is convenient to introduce the following assumptions:

$\left(H_{1}\right)$ There exists a constant $d_{1}>0$ such that

$$
x[g(t, x)-p(t)]>0 \quad \text { for all } t \in \mathbb{R},|x| \geq d_{1} .
$$

$\left(H_{2}\right)$ There exists a constant $d_{2}>0$ such that

$$
x[g(t, x)-p(t)]<0 \quad \text { for all } t \in \mathbb{R},|x| \geq d_{2} .
$$


2. Several lemmas. Let us introduce the auxiliary equation

$$
x^{(n)}+\lambda\left[\sum_{j=1}^{n-1} a_{j} x^{(j)}+g(t, x(t-\tau(t)))\right]=\lambda p(t), \quad \lambda \in(0,1) .
$$

Let

$$
X=\left\{x \in C^{n-1}(\mathbb{R}, \mathbb{R}) \mid x(t+2 \pi)=x(t) \text { for all } t \in \mathbb{R}\right\}
$$

and

$$
Y=\{x \in C(\mathbb{R}, \mathbb{R}) \mid x(t+2 \pi)=x(t) \text { for all } t \in \mathbb{R}\}
$$

be Banach spaces with the norms

$$
\|x\|_{X}=\|x\|=\sum_{j=0}^{n-1}\left|x^{(j)}\right|_{\infty} \quad \text { and } \quad\|x\|_{Y}=|x|_{\infty}=\max _{t \in[0,2 \pi]}|x(t)| .
$$

Define a linear operator $L: D(L) \subset X \rightarrow Y$ by setting

$$
D(L)=\left\{x \in X \mid x^{(n)} \in C(\mathbb{R}, \mathbb{R})\right\}
$$

and for $x \in D(L)$,

$$
L x=x^{(n)} .
$$

We also define a nonlinear operator $N: X \rightarrow Y$ by setting

$$
N x(t)=-\left[\sum_{j=1}^{n-1} a_{j} x^{(j)}+g(t, x(t-\tau(t)))\right]+p(t) .
$$

It is easy to see that

$$
\text { Ker } L=\mathbb{R} \quad \text { and } \quad \operatorname{Im} L=\left\{x \in Y \mid \int_{0}^{2 \pi} x(s) d s=0\right\} .
$$

Thus $L$ is a Fredholm operator with index zero.

Define the continuous projectors $P: X \rightarrow \operatorname{Ker} L$ and $Q: Y \rightarrow Y / \operatorname{Im} L$ by setting

$$
P x(t)=\frac{1}{2 \pi} \int_{0}^{2 \pi} x(s) d s
$$

and

$$
Q x(t)=\frac{1}{2 \pi} \int_{0}^{2 \pi} x(s) d s .
$$

Hence, $\operatorname{Im} P=\operatorname{Ker} L$ and $\operatorname{Ker} Q=\operatorname{Im} L$. Denoting by $L_{P}^{-1}: \operatorname{Im} L \rightarrow D(L) \cap$ Ker $P$ the inverse of $\left.L\right|_{D(L) \cap K e r} P$, one can observe that $L_{P}^{-1}$ is a compact operator. Therefore, $N$ is $L$-compact on $\bar{\Omega}$, where $\Omega$ is an open bounded subset of $X$. 
In view of $(2.2)$ and $(2.2)^{\prime}$, the operator equation

$$
L x=\lambda N x, \quad \lambda \in(0,1),
$$

is equivalent to the auxiliary equation $(2.1)_{\lambda}$.

We now recall the continuation theorem of [8].

Lemma 2.1. Let $X$ and $Y$ be Banach spaces. Suppose that $L: D(L) \subset$ $X \rightarrow Y$ is a Fredholm operator with index zero, and $N: \bar{\Omega} \rightarrow Y$ is L-compact on $\bar{\Omega}$, where $\Omega$ is an open bounded subset of $X$. Moreover, assume that the following conditions are satisfied.

(1) $L x \neq \lambda N x, \forall x \in \partial \Omega \cap D(L), \lambda \in(0,1)$;

(2) $N x \notin \operatorname{Im} L, \forall x \in \partial \Omega \cap \operatorname{Ker} L$;

(3) The Brouwer degree

$$
\operatorname{deg}\{Q N, \Omega \cap \operatorname{Ker} L, 0\} \neq 0 .
$$

Then the equation $L x=N x$ has a solution on $\bar{\Omega} \cap D(L)$.

The following lemmas will be useful to prove our main results in Section 3.

Lemma 2.2. If $x \in C^{2}(\mathbb{R}, \mathbb{R})$ and $x(t+2 \pi)=x(t)$, then

$$
\left|x^{\prime}(t)\right|_{2}^{2} \leq\left|x^{\prime \prime}(t)\right|_{2}^{2} \text {. }
$$

Lemma 2.2 is known as the Wirtinger inequality; for the proof, see [10, $19,20]$.

LEMma 2.3. Let $\left(H_{1}\right)$ or $\left(H_{2}\right)$ hold. If $x(t)$ is a $2 \pi$-periodic solution of $(2.1)_{\lambda}$, then

$$
|x|_{\infty} \leq d+\sqrt{2 \pi}\left|x^{\prime}\right|_{2},
$$

where $d=d_{1}$ or $d_{2}$ according to the case.

Proof. Let $x(t)$ be a $2 \pi$-periodic solution of $(2.1)_{\lambda}$. Integrating $(2.1)_{\lambda}$ from 0 to $2 \pi$, we see that

$$
\int_{0}^{2 \pi}[g(t, x(t-\tau(t)))-p(t)] d t=0 .
$$

Thus, there exists a $\xi \in[0,2 \pi]$ such that

$$
g(\xi, x(\xi-\tau(\xi)))-p(\xi)=0 .
$$

In view of $\left(H_{1}\right)$ or $\left(H_{2}\right)$, we obtain

$$
|x(\xi-\tau(\xi))| \leq d .
$$


Let $\xi=2 m \pi+\bar{\xi}$, where $\bar{\xi} \in[0,2 \pi]$ and $m$ is an integer. Then, using the Schwarz inequality and the relation

$$
|x(t)|=\left|x(\bar{\xi})+\int_{\bar{\xi}}^{t} x^{\prime}(s) d s\right| \leq d+\int_{0}^{2 \pi}\left|x^{\prime}(s)\right| d s, \quad t \in[0,2 \pi],
$$

we have

$$
|x|_{\infty}=\max _{t \in[0,2 \pi]}|x(t)| \leq d+\sqrt{2 \pi}\left|x^{\prime}\right|_{2},
$$

which implies that (2.4) is satisfied.

Lemma 2.4. Assume that $k$ is even, and one of the following conditions is satisfied:

$\left(H_{3}\right) g(t, x)$ is strictly monotone in $x$ and there exists a constant $b$ such that

$0 \leq b<\frac{A_{1}}{2 \pi}, \quad\left|g\left(t, x_{1}\right)-g\left(t, x_{2}\right)\right| \leq b\left|x_{1}-x_{2}\right|$ for all $t, x_{1}, x_{2} \in \mathbb{R} ;$

$\left(H_{4}\right) g(t, x)$ is strictly monotone in $x$ and there exists a constant $b$ such that

$$
0 \leq b<\frac{A_{2}}{2 \pi}, \quad\left|g\left(t, x_{1}\right)-g\left(t, x_{2}\right)\right| \leq b\left|x_{1}-x_{2}\right| \text { for all } t, x_{1}, x_{2} \in \mathbb{R} .
$$

Then (1.4) has at most one $2 \pi$-periodic solution. Then

Proof. Suppose that $x_{1}(t)$ and $x_{2}(t)$ are two $2 \pi$-periodic solutions of (1.4).

$$
\begin{aligned}
\left(x_{1}(t)-x_{2}(t)\right)^{(2 k)}+ & \sum_{j=1}^{2 k-1} a_{j}\left(x_{1}(t)-x_{2}(t)\right)^{(j)} \\
& +\left[g\left(t, x_{1}(t-\tau(t))\right)-g\left(t, x_{2}(t-\tau(t))\right)\right]=0 .
\end{aligned}
$$

Set $Z(t)=x_{1}(t)-x_{2}(t)$. Then $(2.8)$ reads

$$
Z^{(2 k)}(t)+\sum_{j=1}^{2 k-1} a_{j} Z^{(j)}(t)+\left[g\left(t, x_{1}(t-\tau(t))\right)-g\left(t, x_{2}(t-\tau(t))\right)\right]=0 .
$$

Integrating (2.9) from 0 to $2 \pi$, we have

$$
\int_{0}^{2 \pi}\left[g\left(t, x_{1}(t-\tau(t))\right)-g\left(t, x_{2}(t-\tau(t))\right)\right] d t=0 .
$$

Thus, in view of the integral mean value theorem, there exists a constant $\gamma \in[0,2 \pi]$ such that

$$
g\left(\gamma, x_{1}(\gamma-\tau(\gamma))\right)-g\left(\gamma, x_{2}(\gamma-\tau(\gamma))\right)=0 .
$$


Let $\gamma-\tau(\gamma)=m_{1} 2 \pi+\widetilde{\gamma}$, where $\widetilde{\gamma} \in[0,2 \pi]$ and $m_{1}$ is an integer. Then (2.10), together with $\left(H_{3}\right)$ (or $\left.\left(H_{4}\right)\right)$, implies that

$$
Z(\widetilde{\gamma})=x_{1}(\widetilde{\gamma})-x_{2}(\widetilde{\gamma})=x_{1}(\gamma-\tau(\gamma))-x_{2}(\gamma-\tau(\gamma))=0 .
$$

Hence,

$$
|Z(t)|=\left|Z(\widetilde{\gamma})+\int_{\widetilde{\gamma}}^{t} Z^{\prime}(s) d s\right| \leq \int_{0}^{2 \pi}\left|Z^{\prime}(s)\right| d s, \quad t \in[0,2 \pi]
$$

and

$$
|Z|_{\infty} \leq \sqrt{2 \pi}\left|Z^{\prime}\right|_{2}
$$

Now we consider two cases.

CASE (i): $\left(H_{3}\right)$ holds. Multiplying $(2.9)$ by $Z^{(2 k)}(t)$ and then integrating from 0 to $2 \pi$, in view of $(2.3),(2.9)$ and the Schwarz inequality, we have

$$
\begin{aligned}
& A_{1}\left|Z^{(2 k)}\right|_{2}^{2}=A_{1} \int_{0}^{2 \pi}\left|Z^{(2 k)}(t)\right|^{2} d t \\
= & \left(1-a_{2(k-1)}^{+}-\left|a_{2(k-2)}\right|-\cdots-\left|a_{4}\right|-a_{2}^{+}\right) \int_{0}^{2 \pi}\left|Z^{(2 k)}(t)\right|^{2} d t \\
\leq & \int_{0}^{2 \pi}\left|Z^{(2 k)}(t)\right|^{2} d t+\int_{0}^{2 \pi}\left[-a_{2(k-1)}^{+}\left|Z^{(2 k-1)}(t)\right|^{2}-\left|a_{2(k-2)}\right|\left|Z^{(2 k-2)}(t)\right|^{2}\right. \\
& \left.-\cdots-\left|a_{4}\right|\left|Z^{(k+2)}(t)\right|^{2}-a_{2}^{+}\left|Z^{(k+1)}(t)\right|^{2}\right] d t \\
\leq & \int_{0}^{2 \pi}\left|Z^{(2 k)}(t)\right|^{2} d t+\int_{0}^{2 \pi} \sum_{j=1}^{2 k-1} a_{j} Z^{(j)}(t) Z^{(2 k)}(t) d t \\
= & -\int_{0}^{2 \pi}\left[g\left(t, x_{1}(t-\tau(t))\right)-g\left(t, x_{2}(t-\tau(t))\right)\right] Z^{(2 k)}(t) d t \\
\leq & b \int_{0}^{2 \pi}\left|x_{1}(t-\tau(t))-x_{2}(t-\tau(t))\right|\left|Z^{(2 k)}(t)\right| d t .
\end{aligned}
$$

From (2.3), (2.12) and the Schwarz inequality, (2.13) implies that

$$
\begin{aligned}
A_{1}\left|Z^{(2 k)}\right|_{2}^{2} & \leq b|Z|_{\infty} \sqrt{2 \pi}\left|Z^{(2 k)}\right|_{2} \leq b \sqrt{2 \pi}\left|Z^{\prime}\right|_{2} \sqrt{2 \pi}\left|Z^{(2 k)}\right|_{2} \\
& \leq 2 \pi b\left|Z^{(2 k)}\right|_{2}^{2} .
\end{aligned}
$$

Since $Z(t), Z^{\prime}(t), \ldots, Z^{(2 k)}(t)$ are $2 \pi$-periodic and continuous functions, in view of $\left(H_{3}\right),(2.11)$ and $(2.14)$, we have

$$
Z(t) \equiv Z^{\prime}(t) \equiv \cdots \equiv Z^{(2 k)}(t) \equiv 0 \quad \text { for all } t \in \mathbb{R} .
$$

Thus, $x_{1}(t) \equiv x_{2}(t)$ for all $t \in \mathbb{R}$. 
CASE (ii): $\left(H_{4}\right)$ holds. Multiplying $(2.9)$ by $Z^{(2 k-1)}(t)$ and then integrating from 0 to $2 \pi$, in view of $(2.3),(2.9),(2.12)$ and the Schwarz inequality, we get

$$
A_{2}\left|Z^{(2 k-1)}\right|_{2}^{2}=A_{2} \int_{0}^{2 \pi}\left|Z^{(2 k-1)}(t)\right|^{2} d t
$$

$$
\begin{aligned}
= & \left(a_{2 k-1}-a_{2 k-3}^{+}-\left|a_{2 k-5}\right|-\cdots-\left|a_{3}\right|-a_{1}^{+}\right) \int_{0}^{2 \pi}\left|Z^{(2 k-1)}(t)\right|^{2} d t \\
\leq & a_{2 k-1} \int_{0}^{2 \pi}\left|Z^{(2 k-1)}(t)\right|^{2} d t+\int_{0}^{2 \pi}\left[-a_{2 k-3}^{+}\left|Z^{(2 k-2)}(t)\right|^{2}-\left|a_{2 k-5}\right|\left|Z^{(2 k-3)}(t)\right|^{2}\right. \\
& \left.-\cdots-\left|a_{3}\right|\left|Z^{(k+1)}(t)\right|^{2}-a_{1}^{+}\left|Z^{(k)}(t)\right|^{2}\right] d t
\end{aligned}
$$$$
\leq a_{2 k-1} \int_{0}^{2 \pi}\left|Z^{(2 k-1)}(t)\right|^{2} d t+\int_{0}^{2 \pi} \sum_{j=1}^{2 k-2} a_{j} Z^{(j)}(t) Z^{(2 k-1)}(t) d t
$$$$
=-\int_{0}^{2 \pi}\left[g\left(t, x_{1}(t-\tau(t))\right)-g\left(t, x_{2}(t-\tau(t))\right)\right] Z^{(2 k-1)}(t) d t
$$

$$
\leq b \int_{0}^{2 \pi}\left|x_{1}(t-\tau(t))-x_{2}(t-\tau(t))\right|\left|Z^{(2 k-1)}(t)\right| d t \leq 2 \pi b\left|Z^{(2 k-1)}\right|_{2}^{2} .
$$

From $(2.11)$ and $\left(H_{4}\right),(2.15)$ implies that

$$
Z(t) \equiv Z^{\prime}(t) \equiv \cdots \equiv Z^{(2 k-1)}(t) \equiv 0 \quad \text { for all } t \in \mathbb{R} .
$$

Hence, $x_{1}(t) \equiv x_{2}(t)$ for all $t \in \mathbb{R}$. The proof of Lemma 2.4 is now complete.

In a similar fashion we can show the following:

Lemma 2.5. Assume that $k$ is odd, and one of the following conditions is satisfied:

$\left(\widetilde{H}_{3}\right) g(t, x)$ is strictly monotone in $x$ and there exists a constant $b$ such that

$0 \leq b<\frac{\bar{A}_{1}}{2 \pi}, \quad\left|g\left(t, x_{1}\right)-g\left(t, x_{2}\right)\right| \leq b\left|x_{1}-x_{2}\right|$ for all $t, x_{1}, x_{2} \in \mathbb{R} ;$

$\left(\widetilde{H}_{4}\right) g(t, x)$ is strictly monotone in $x$ and there exists a constant $b$ such that

$0 \leq b<\frac{\bar{A}_{2}}{2 \pi}, \quad\left|g\left(t, x_{1}\right)-g\left(t, x_{2}\right)\right| \leq b\left|x_{1}-x_{2}\right|$ for all $t, x_{1}, x_{2} \in \mathbb{R}$.

Then (1.4) has at most one $2 \pi$-periodic solution. 


\section{Main results}

Theorem 3.1. Let $\left(H_{1}\right)$ or $\left(H_{2}\right)$ hold. Assume that $k$ is even, and either $\left(H_{3}\right)$ or $\left(H_{4}\right)$ is satisfied. Then (1.4) has a unique $2 \pi$-periodic solution.

Proof. By Lemma 2.4, we only have to prove the existence. To do this, we shall apply Lemma 2.1. First, we claim that all $2 \pi$-periodic solutions of $(2.1)_{\lambda}$ are bounded. We consider two cases.

CASE (1): $\left(H_{3}\right)$ holds. Let $x(t)$ be a $2 \pi$-periodic solution of $(2.1)_{\lambda}$. Multiplying $(2.1)_{\lambda}$ by $x^{(2 k)}(t)$ and then integrating from 0 to $2 \pi$, in view of (2.3), (2.4), $\left(H_{3}\right)$ and the Schwarz inequality, we have

$$
\begin{aligned}
& A_{1}\left|x^{(2 k)}\right|_{2}^{2}=\left(1-a_{2(k-1)}^{+}-\left|a_{2(k-2)}\right|-\cdots-\left|a_{4}\right|-a_{2}^{+}\right) \int_{0}^{2 \pi}\left|x^{(2 k)}(t)\right|^{2} d t \\
& \leq \int_{0}^{2 \pi}\left|x^{(2 k)}(t)\right|^{2} d t+\int_{0}^{2 \pi} \lambda\left[-a_{2(k-1)}^{+}\left|x^{(2 k-1)}(t)\right|^{2}-\left|a_{2(k-2)}\right|\left|x^{(2 k-2)}(t)\right|^{2}\right. \\
& \left.-\cdots-\left|a_{4}\right|\left|x^{(k+2)}(t)\right|^{2}-a_{2}^{+}\left|x^{(k+1)}(t)\right|^{2}\right] d t \\
& \leq \int_{0}^{2 \pi}\left|x^{(2 k)}(t)\right|^{2} d t+\lambda \int_{0}^{2 \pi} \sum_{j=1}^{2 k-1} a_{j} x^{(j)}(t) x^{(2 k)}(t) d t \\
& =-\int_{0}^{2 \pi} g(t, x(t-\tau(t))) x^{(2 k)}(t) d t+\int_{0}^{2 \pi} p(t) x^{(2 k)}(t) d t \\
& \leq \int_{0}^{2 \pi}[|g(t, x(t-\tau(t)))-g(t, 0)|+|g(t, 0)|]\left|x^{(2 k)}(t)\right| d t \\
& +\int_{0}^{2 \pi}|p(t)|\left|x^{(2 k)}(t)\right| d t \\
& \leq b \int_{0}^{2 \pi}|x(t-\tau(t))|\left|x^{(2 k)}(t)\right| d t+\int_{0}^{2 \pi}|g(t, 0)|\left|x^{(2 k)}(t)\right| d t \\
& +\int_{0}^{2 \pi}|p(t)|\left|x^{(2 k)}(t)\right| d t \\
& \leq b \sqrt{2 \pi}\left|x^{\prime}\right|_{2} \sqrt{2 \pi}\left|x^{(2 k)}\right|_{2}+\left[b d+\max _{0 \leq t \leq 2 \pi}|g(t, 0)|+|p|_{\infty}\right] \sqrt{2 \pi}\left|x^{(2 k)}\right|_{2} \\
& \leq 2 \pi b\left|x^{(2 k)}\right|_{2}^{2}+\left[b d+\max _{0 \leq t \leq 2 \pi}|g(t, 0)|+|p|_{\infty}\right] \sqrt{2 \pi}\left|x^{(2 k)}\right|_{2} .
\end{aligned}
$$

Since $b<A_{1} / 2 \pi,(2.3),(2.4)$ and (3.1) imply that there exists a constant 
$D_{1}>0$ such that

$$
\begin{aligned}
& \left|x^{(j)}\right|_{2} \leq\left|x^{(2 k)}\right|_{2} \leq D_{1}, \quad j=1, \ldots, 2 k-1, \\
& |x|_{\infty} \leq d+\sqrt{2 \pi}\left|x^{\prime}\right|_{2} \leq D_{1} .
\end{aligned}
$$

For $j=1, \ldots, 2 k-1$, noting that $x^{(j)}(t)$ are $2 \pi$-periodic, there exists a $T_{j} \in(0,2 \pi)$ such that $x^{(j+1)}\left(T_{j}\right)=0$. Therefore,

$$
\begin{aligned}
\left|x^{(j)}(t)\right| & =\left|\int_{T_{j}}^{t} x^{(j+1)}(s) d s\right| \leq \sqrt{2 \pi}\left(\int_{0}^{2 \pi}\left|x^{(j+1)}(s)\right|^{2} d s\right)^{1 / 2} \\
& \leq \sqrt{2 \pi}\left|x^{(j+1)}\right|_{2} \leq \sqrt{2 \pi} D_{1} .
\end{aligned}
$$

Therefore, for all possible $2 \pi$-periodic solutions $x(t)$ of $(2.1)_{\lambda}$, there exists a constant $M_{1}$ such that

$$
\|x\|=\sum_{j=0}^{2 k-1}\left|x^{(j)}\right|_{\infty}<M_{1},
$$

with $M_{1}>0$ independent of $\lambda$.

CASE $(2)$ : $\left(H_{4}\right)$ holds. Let $x(t)$ be a $2 \pi$-periodic solution of $(2.1)_{\lambda}$. Multiplying $(2.1)_{\lambda}$ by $x^{(2 k-1)}(t)$ and then integrating from 0 to $2 \pi$, by $\left(H_{4}\right)$, (2.3), (2.4) and the Schwarz inequality, we have

$$
\begin{aligned}
= & \left(a_{2 k-1}-a_{2 k-3}^{+}-\left|a_{2 k-5}\right|-\cdots-\left|a_{3}\right|-a_{1}^{+}\right) \int_{0}^{2 \pi}\left|x^{(2 k-1)}(t)\right|^{2} d t \\
\leq & a_{2 k-1} \int_{0}^{2 \pi}\left|x^{(2 k-1)}(t)\right|^{2} d t+\int_{0}^{2 \pi}\left[-a_{2 k-3}^{+}\left|x^{(2(k-1)}(t)\right|^{2}-\left|a_{2 k-5}\right|\left|x^{(2 k-3)}(t)\right|^{2}\right. \\
& \left.-\cdots-\left|a_{3}\right|\left|x^{(k+1)}(t)\right|^{2}-a_{1}^{+}\left|x^{(k)}(t)\right|^{2}\right] d t \\
\leq & a_{2 k-1} \int_{0}^{2 \pi}\left|x^{(2 k-1)}(t)\right|^{2} d t+\int_{0}^{2 \pi} \sum_{j=1}^{2 k-1} a_{j} x^{(j)}(t) x^{(2 k-1)}(t) d t \\
= & -\int_{0}^{2 \pi} g(t, x(t-\tau(t))) x^{(2 k-1)}(t) d t+\int_{0}^{2 \pi} p(t) x^{(2 k-1)}(t) d t \\
\leq & b \int_{0}^{2 \pi}|x(t-\tau(t))|\left|x^{(2 k-1)}(t)\right| d t+\int_{0}^{2 \pi}|g(t, 0)|\left|x^{(2 k-1)}(t)\right| d t \\
& +\int_{0}^{2 \pi}|p(t)|\left|x^{(2 k-1)}(t)\right| d t \\
\leq & 2 \pi b\left|x^{\prime}\right|_{2}\left|x^{(2 k-1)}\right|_{2}+\left[b d+\max _{0 \leq t \leq 2 \pi}|g(t, 0)|+|p|_{\infty}\right] \sqrt{2 \pi}\left|x^{(2 k-1)}\right|_{2} \\
\leq & 2 \pi b\left|x^{(2 k-1)}\right|_{2}^{2}+\left[b d+\max _{0 \leq t \leq 2 \pi}|g(t, 0)|+|p|_{\infty}\right] \sqrt{2 \pi}\left|x^{(2 k-1)}\right|_{2} .
\end{aligned}
$$


Since $b<A_{2} / 2 \pi,(2.3),(2.4)$ and (3.5) imply that there exists a constant $D_{2}>0$ such that

$$
\begin{aligned}
& \left|x^{(j)}\right|_{2} \leq\left|x^{(2 k-1)}\right|_{2} \leq D_{2}, \quad j=1,2, \ldots, 2 k-2, \\
& |x|_{\infty} \leq d+\sqrt{2 \pi}\left|x^{\prime}\right|_{2} \leq D_{2} .
\end{aligned}
$$

From $(2.1)_{\lambda},(3.3)$ and (3.6), we obtain

$$
\begin{aligned}
\left|x^{(2 k-1)}(t)\right| & =\left|\int_{T_{2 k-1}}^{t} x^{(2 k)}(s) d s\right| \\
& \leq \int_{0}^{2 \pi}\left|-\left[\sum_{j=1}^{2 k-1} a_{j} x^{(j)}+g(t, x(t-\tau(t)))\right]+p(t)\right| d s \\
& \leq \sum_{j=1}^{n-1}\left|a_{j}\right| \sqrt{2 \pi} D_{2}+2 \pi\left[\max _{t \in R,|x| \leq D_{2}}|g(t, x)|+|p|_{\infty}\right] \\
& =: \bar{D}_{1},
\end{aligned}
$$

which, together with (3.6), implies that (3.4) also holds.

If $x \in \Omega_{1}=\{x \in \operatorname{Ker} L \cap X \mid N x \in \operatorname{Im} L\}$, then there exists a constant $M_{2}$ such that

$$
x(t) \equiv M_{2}, \quad \int_{0}^{2 \pi}\left[g\left(t, M_{2}\right)-p(t)\right] d t=0 .
$$

Thus,

$$
|x(t)| \equiv\left|M_{2}\right|<d \quad \text { for all } x \in \Omega_{1} .
$$

Let $M=M_{1}+d$. Set

$$
\Omega=\left\{\left.x \in X\left|\|x\|=\sum_{j=0}^{2 k-1}\right| x^{(j)}\right|_{\infty}<M\right\} .
$$

Since $N$ is $L$-compact on $\bar{\Omega}$, it is easy to see from (3.4), (3.8) and (3.9) that the conditions (1) and (2) in Lemma 2.1 hold.

Furthermore, define continuous functions $\Psi_{1}(x, \mu)$ and $\Psi_{2}(x, \mu)$ by setting, for $x \in \mathbb{R}$ and $\mu \in[0,1]$,

$$
\begin{aligned}
& \Psi_{1}(x, \mu)=-(1-\mu) x-\mu \cdot \frac{1}{2 \pi} \int_{0}^{2 \pi}[g(t, x)-p(t)] d t, \\
& \Psi_{2}(x, \mu)=(1-\mu) x-\mu \cdot \frac{1}{2 \pi} \int_{0}^{2 \pi}[g(t, x)-p(t)] d t .
\end{aligned}
$$


If $\left(H_{1}\right)$ holds, then

$$
x \Psi_{1}(x, \mu) \neq 0 \quad \text { for all } x \in \partial \Omega \cap \operatorname{Ker} L .
$$

Hence, using the homotopy invariance theorem, we have

$$
\begin{aligned}
\operatorname{deg}\{Q N, \Omega \cap \operatorname{Ker} L, 0\} & =\operatorname{deg}\left\{-\frac{1}{2 \pi} \int_{0}^{2 \pi}[g(t, x)-p(t)] d t, \Omega \cap \operatorname{Ker} L, 0\right\} \\
& =\operatorname{deg}\{-x, \Omega \cap \operatorname{Ker} L, 0\} \neq 0 .
\end{aligned}
$$

If $\left(H_{2}\right)$ holds, then

$$
x \Psi_{2}(x, \mu) \neq 0 \quad \text { for all } x \in \partial \Omega \cap \operatorname{Ker} L .
$$

Hence, using the homotopy invariance theorem, we obtain

$$
\begin{aligned}
\operatorname{deg}\{Q N, \Omega \cap \operatorname{Ker} L, 0\} & =\operatorname{deg}\left\{-\frac{1}{2 \pi} \int_{0}^{2 \pi}[g(t, x)-p(t)] d t, \Omega \cap \operatorname{Ker} L, 0\right\} \\
& =\operatorname{deg}\{x, \Omega \cap \operatorname{Ker} L, 0\} \neq 0 .
\end{aligned}
$$

In view of the above discussion, we conclude from Lemma 2.1 that Theorem 3.1 is proved.

In view of Lemma 2.5, a similar argument leads to

TheOREM 3.2. Let $\left(H_{1}\right)$ or $\left(H_{2}\right)$ hold. Assume that $k$ is odd, and either $\left(\widetilde{H}_{3}\right)$ or $\left(\widetilde{H}_{4}\right)$ is satisfied. Then (1.4) has a unique $2 \pi$-periodic solution.

We are now in a position to establish the existence and uniqueness of $2 \pi$-periodic solutions of equation (1.5). Similarly to the proof of Theorems 3.1 and 3.2 , one can prove the following results.

Theorem 3.3. Let $\left(H_{1}\right)$ or $\left(H_{2}\right)$ hold. Assume that $k$ is even, and one of the following conditions is satisfied:

$\left(H_{5}\right) g(t, x)$ is strictly monotone in $x$ and there exists a constant $b$ such that

$0 \leq b<\frac{A_{3}}{2 \pi}, \quad\left|g\left(t, x_{1}\right)-g\left(t, x_{2}\right)\right| \leq b\left|x_{1}-x_{2}\right|$ for all $t, x_{1}, x_{2} \in \mathbb{R} ;$

$\left(H_{6}\right) g(t, x)$ is strictly monotone in $x$ and there exists a constant $b$ such that

$0 \leq b<\frac{A_{4}}{2 \pi}, \quad\left|g\left(t, x_{1}\right)-g\left(t, x_{2}\right)\right| \leq b\left|x_{1}-x_{2}\right|$ for all $t, x_{1}, x_{2} \in \mathbb{R}$.

Then (1.5) has a unique $2 \pi$-periodic solution.

Theorem 3.4. Let $\left(H_{1}\right)$ or $\left(H_{2}\right)$ hold. Assume that $k$ is odd, and one of the following conditions is satisfied: 
$\left(\widetilde{H}_{5}\right) g(t, x)$ is strictly monotone in $x$ and there exists a constant $b$ such that

$0 \leq b<\frac{\bar{A}_{3}}{2 \pi}, \quad\left|g\left(t, x_{1}\right)-g\left(t, x_{2}\right)\right| \leq b\left|x_{1}-x_{2}\right|$ for all $t, x_{1}, x_{2} \in \mathbb{R}$

$\left(\widetilde{H}_{6}\right) g(t, x)$ is strictly monotone in $x$ and there exists a constant $b$ such that

$0 \leq b<\frac{\bar{A}_{4}}{2 \pi}, \quad\left|g\left(t, x_{1}\right)-g\left(t, x_{2}\right)\right| \leq b\left|x_{1}-x_{2}\right|$ for all $t, x_{1}, x_{2} \in \mathbb{R}$.

Then (1.5) has a unique $2 \pi$-periodic solution.

\section{Example and remark}

ExAMPLE 4.1. Let $g(t, x(t-\tau(t)))=-\frac{1}{3} x\left(t-30 e^{\sin t}\right) e^{\sin t}$ and $p(t)=$ $2 \cos t$. Then the equation

$$
x^{(6)}+100 x^{(5)}+x^{(4)}-10 x^{(3)}+20 x^{\prime \prime}-6 x^{\prime}+g(t, x(t-\tau(t)))=e(t)
$$

has a unique $2 \pi$-periodic solution.

Proof. It is straightforward to check that the assumptions $\left(H_{2}\right)$ and $\left(\widetilde{H}_{4}\right)$ are satisfied. Therefore, by Theorem 3.2, equation (4.1) has a unique $2 \pi$ periodic solution.

REMARK 4.1. As in $[1,2,5,6,12-14]$, the papers $[16,17]$ only study the existence of periodic solutions. Therefore, the results in $[1-6,7,9,11-$ 21 ] and the references therein cannot be applied to show the uniqueness of $2 \pi$-periodic solutions of equation (4.1). This implies that the results of this paper are essentially new.

\section{References}

[1] K. J. Brown and S. S. Lin, Periodically perturbed conservative systems and a global inverse function theorem, Nonlinear Anal. 4 (1979), 193-201.

[2] T. A. Burton, Stability and Periodic Solutions of Ordinary and Functional Differential Equations, Academic Press, Orlando, FL, 1985.

[3] A. Cañada and P. Martínez-Amores, Solvability of some operator equations and periodic solutions of nonlinear functional-differential equations, J. Differential Equations 49 (1983), 415-429.

[4] J. D. Cao and G. M. He, Periodic solutions for higher-order neutral differential equations with several delays, Comput. Math. Appl. 48 (2004), 1491-1503.

[5] F. Z. Cong, Periodic solutions for $2 k$ th order ordinary differential equations with nonresonance, Nonlinear Anal. 32 (1997), 787-793.

[6] F. Z. Cong, Q. D. Huang and S. Y. Shi, Existence and uniqueness of periodic solutions for $(2 n+1)$ th order differential equations, J. Math. Anal. Appl. 241 (2000), $1-9$.

[7] S. Fučik and J. Mawhin, Periodic solutions of some nonlinear differential equations of higher order, Časopis Pěst. Mat. 100 (1975), 276-283. 
[8] R. E. Gaines and J. Mawhin, Coincidence Degree and Nonlinear Differential Equations, Lecture Notes in Math. 568, Springer, 1977.

[9] R. Grafton, Periodic solutions of certain Liénard equations with delay, J. Differential Equations 11 (1972), 519-527.

[10] G. H. Hardy, J. E. Littlewood and G. Pólya, Inequalities, Cambridge Univ. Press, London, 1964.

[11] R. Iannacci and M. N. Nkashama, On periodic solutions of forced second order differential equations with deviating arguments, in: Lecture Notes in Math. 1151, Springer, 1984, 224-232.

[12] A. C. Lazer, Application of a lemma on bilinear forms to a problem in nonlinear oscillations, Proc. Amer. Math. Soc. 33 (1972), 89-94.

[13] W. G. Li, Periodic solutions for $2 k$ th order ordinary differential equations with resonance, J. Math. Anal. Appl. 259 (2001), 157-167.

[14] Y. Li and H. Z. Wang, Periodic solutions of higher-order Duffing equations, Appl. Math. J. Chinese Univ. 6 (1991), 407-412 (in Chinese).

[15] B. W. Liu and L. H. Huang, Periodic solutions for nonlinear nth order differential equations with delays, J. Math. Anal. Appl. 313 (2006), 700-716.

[16] W. B. Liu and Y. Li, Existence of periodic solutions for higher-order Duffing equations, Acta Math. Sinica 46 (2003), 49-56 (in Chinese).

[17] Z. L. Liu, Periodic solutions for nonlinear nth order ordinary differential equations, J. Math. Anal. Appl. 204 (1996), 46-64.

[18] S. P. Lu and W. G. Ge, Periodic solutions for a kind of Liénard equations with a deviating argument, ibid. 249 (2004), 231-243.

[19] J. Mawhin, Degré topologique et solutions périodiques des systèmes différentiels non linéaires, Bull. Soc. Roy. Sci. Liège 38 (1969), 308-398.

[20] - An extension of a theorem of A. C. Lazer on forced nonlinear oscillations, J. Math. Anal. Appl. 40 (1972), 20-29.

[21] - , L $L_{2}$-estimates and periodic solutions of some nonlinear differential equations, Boll. Un. Mat. Ital. (4) 10 (1974), 341-354.

National Science Library

Chinese Academy of Sciences

Beijing 100190, P.R. China

E-mail: sweiwen@amss.ac.cn

Editorial Department

Journal of Hunan University

Changsha, Hunan, 410082, P.R. China

E-mail: yalihd1969@yahoo.cn
Department of Mathematics

Shaoyang University

Shaoyang, Hunan, 422000, P.R. China

E-mail: fuxingzhang2006@163.com

Received 11.3.2007

and in final form 19.3.2008 